\title{
Endoscopic vacuum therapy in the management of anastomotic insufficiency after pancreatico- duodenectomy
}

A 68-year-old patient with a pT3pN2 duodenal adenocarcinoma underwent pancreaticoduodenectomy. Because the jejunal loop was anastomosed with a previous gastroenterostomy, endoscopic examination of the pancreatic and biliary anastomoses was easily feasible. On the 5 th postoperative day, the patient developed septic shock and multiorgan failure; bilious secretions were noted coming from the abdominal drains. An immediate revision laparotomy was performed, at which generalized peritonitis was found. It was associated with total dehiscence of the biliojejunal anastomosis and subtotal dehiscence of the pancreaticojejunal anastomosis. Operative reconstruction of the anastomoses was impossible. Four laparotomies with lavage of the peritoneal cavity were scheduled at 48-hour intervals. During this time, an acute hepatic artery bleed caused by septic erosion occurred.

During the third laparotomy, intracavitary vacuum therapy was also started. This consists of a drainage system made by a size-adjusted, open-pore polyurethane sponge (Endo-SPONGE; B.Braun, Melsungen, Germany) that is fixed to the distal end of a gastric tube (Ventrol, $14 \mathrm{Fr} \times 120 \mathrm{~cm}$; Covidien-Nellcor, Boulder, Colorado, USA) and connected to an electronic vacuum device that generates 125-mmHg continuous negative pressure (V.A.C. Freedom; KCI, San Antonio, Texas, USA). We differentiate between intracavitary and intraluminal vacuum therapy and have described both therapeutic modalities and their clinical results in a previous publication [1].

A small percutaneous vacuum drain was placed directly onto the region of subhepatic anastomotic insufficiency. To promote compartmentalization of the septic focus, colonic mesentery was placed ventral to the vacuum sponge drainage. Biliary and pancreatic secretions were evacuated mainly by the vacuum drainage system but also partly by additional local gravity drainage. These measures allowed us to compartmentalize the septic focus and effectively drain the biliary and pancreatic secretion to stop further intra-abdominal inflammation.

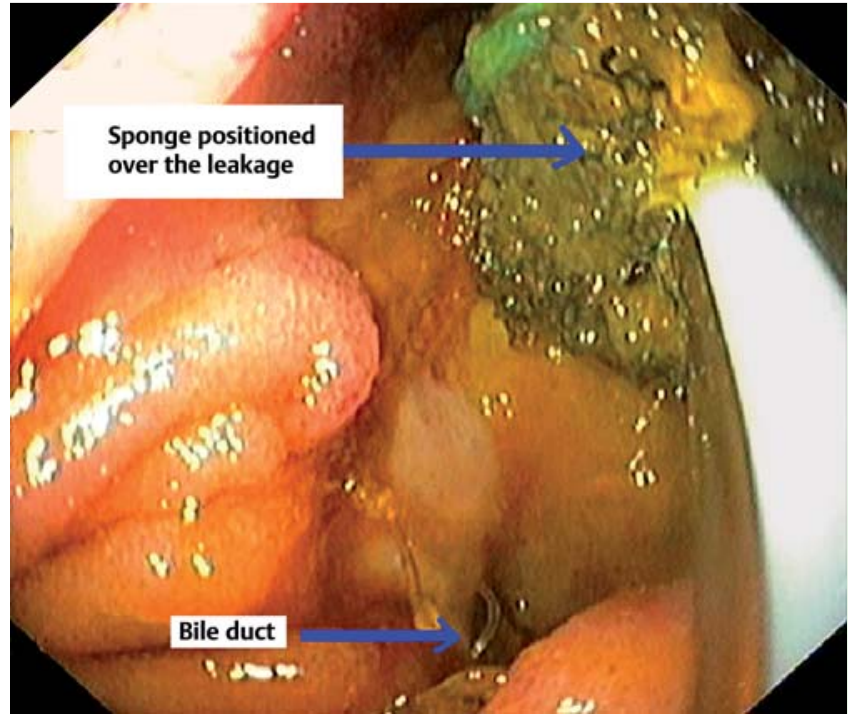

Fig. 1 Endoscopic view showing the sponge connected to the vacuum drainage system in position over the area of leakage.
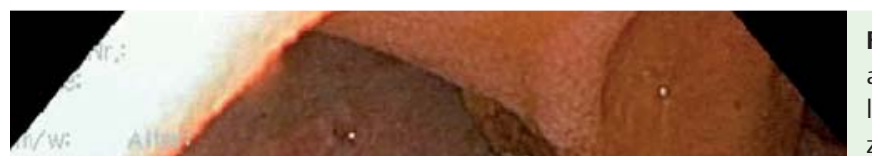

Fig. 2 Endoscopic appearance 4 weeks later showing normalization of the anatomic situation.

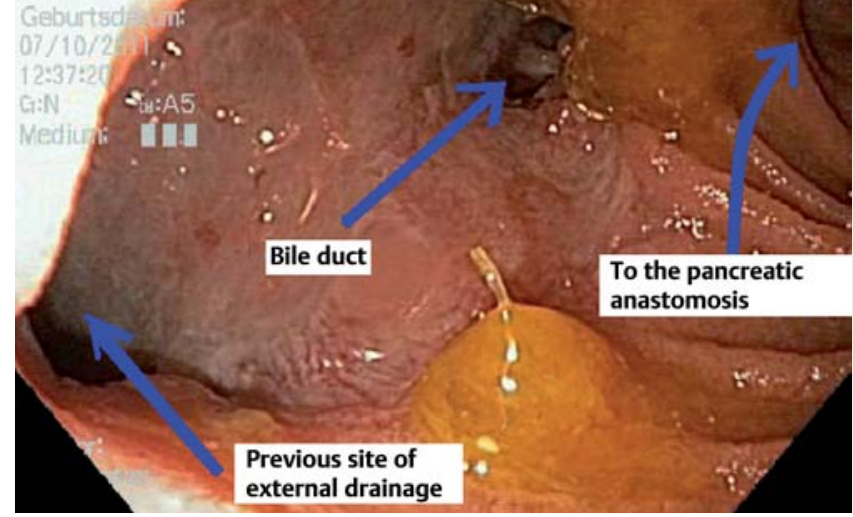

The next aim was to functionally reconstruct the anastomoses. We used the principle of intraluminal vacuum therapy and endoscopically placed a vacuum drainage system via the jejunal loop that was covering the dehiscent pancreaticojejunal and biliojejunal anastomoses. Thus, it was possible to drain the digestive juices into the jejunal lumen and the additional gravity drainage showed a steady decrease in flow rate.

Subsequent adjustments were made to the endoscopic vacuum drainage system solely by endoscopic means using the procedure described in our previous publications [2]. The sponge tip was placed in contact with the bile duct and pancreatic tail through the jejunal defects, simultaneously draining both ( $\bullet$ Fig. 1 ). In the course of the vacuum therapy, the wound cavity developed stable granulation tissue and gradually reduced in size. It was possible to perform the six changes of the sponge by endoscopic means on the intensive care unit, without the requirement for a further laparotomy.

After two periods of treatment over a total of 28 days, endoscopic vacuum therapy was discontinued. The markers of systemic inflammation had returned to normal and pancreatic and biliary secretions were draining into the jejunal loop without leak- 
age. A follow-up endoscopy 4 weeks later showed normalization of the anatomic situation with the bile and pancreatic ducts firmly connected to the jejunal loop (๑ Fig. 2). There was no cutaneous fistula. Parenteral nutrition was necessary in the beginning because of the sepsis and the need for revision laparotomies, but during the endoscopic vacuum therapy, enteral nutrition via a gastric tube was possible. Immediately after cessation of the vacuum therapy, a step-by-step return to a normal diet was initiated, and this was well tolerated by the patient.

Anastomotic insufficiency after pancreaticoduodenectomy has a very high mortality rate and is very difficult to treat. Because of the severe inflammation with generalized peritonitis, surgical intervention and diversion of the irritant secretions through passive drainage systems was indispensable in our case. Endoscopic vacuum therapy provided us with an effective method to compartmentalize the septic focus by effective drainage of the irritant biliary and pancreatic secretions.
Furthermore, by directing these fluids into the jejunal loop, intraluminal vacuum therapy led to the reconstruction of normal anastomotic anatomy.

Therefore, a combination of approaches enabled us to scale down the treatment from traumatizing operative procedures to a minimally invasive therapy without losing the full visualization and control of the inner wound that is associated with open surgery.

To our knowledge, this is the first report of successful treatment for biliary and pancreatic anastomotic insufficiency after pancreaticoduodenal resection with endoscopic vacuum therapy. The combination of operative and endoscopic treatment strategies opens new possibilities for successful tools in the management of postoperative complications.

Endoscopy_UCTN_Code_TTT_1AO_2AI

Competing interests: None

\section{G. Loske, T. Strauss, B. Riefel, C. T. Mueller, T. Schorsch}

Department of Surgery, Katholisches Marienkrankenhaus, Hamburg, Germany

\section{References}

1 Loske G, Schorsch T, Muller C. Intraluminal and intracavitary vacuum therapy for esophageal leakage: a new endoscopic minimally invasive approach. Endoscopy 2011; 43: $540-544$

2 Loske G, Schorsch T, Muller C. Endoscopic vacuum sponge therapy for esophageal defects. Surg Endosc 2010; 24: 2531 -2535

Bibliography

DOI http://dx.doi.org/

10.1055/s-0031-1291698

Endoscopy 2012; 44: E94-E95

(C) Georg Thieme Verlag KG

Stuttgart · New York

ISSN 0013-726X

Corresponding author

G. Loske, MD

Department of Surgery

Katholisches Marienkrankenhaus

Alfredstrasse 9

22087 Hamburg

Germany

Phone: +49-40-25461402

loske.chir@marienkrankenhaus.org 